\title{
Thermal Annealing of CdMnTe Material Being Developed for Nuclear Radiation Detection Applications
}

\author{
Aaron L. Adams ${ }^{1}$, Ezekiel O. Agbalagba ${ }^{2}$, Julius O. Jow ${ }^{3}$, John G. Mwathi ${ }^{3}$, \\ Alexander A. Egarievwe ${ }^{1}$, Wing Chan ${ }^{3}$, Matthew C. Dowdell ${ }^{3}$, Utpal N. Roy ${ }^{4}$, \\ Stephen U. Egarievwe ${ }^{3}$ \\ ${ }^{I}$ (Department of Mechanical and Civil Engineering, Alabama A\&M University, Normal, AL, USA) \\ ${ }^{2}$ (Department of Physics, Federal University of Petroleum Resources, Effurun, Delta State, Nigeria) \\ ${ }_{3}^{3}$ (Nuclear Engineering and Radiological Science Center, Alabama A\&M University, Normal, AL, USA) \\ ${ }^{4}$ (Department of Nonproliferation and National Security, Brookhaven National Laboratory, Upton, NY, USA)
}

\begin{abstract}
Cadmium manganese telluride (CdMnTe) is one of the semiconductor materials with potential applications at room-temperature for nuclear and radiological detection. CdMnTe crystals grown by Bridgman technique are prone to tellurium inclusions and related defects that limit their performance as X-rays and gamma-rays detectors. The major reason for this is that they are grown in a tellurium-rich environment. These defects could trap charges that are generated by X-rays and gamma rays thereby degrading the charge transport properties of the detectors and reducing their carrier lifetime. This in turn leads to poor performance by the detector. One of the solutions to this problem is post-growth thermal annealing. In this paper we present experimental results of annealing a CdMnTe wafer at $720^{\circ} \mathrm{C}$ and in cadmium vapor. The CdMnTe wafer and cadmium were sealed in a quartz ampoule at a vacuum of $10^{-5}$ mbar. We used a three-zone furnace that enabled us to adjust the three heating elements to get a flat region of $720^{\circ} \mathrm{C}$ in the temperature profile where the wafer was annealed. Infrared transmission microscopy showed changes to the sizes and positions of the tellurium inclusions. There are reductions in the dimensions of the medium-size Te inclusions. Some Te inclusions were completely eliminated while others broke up to form much smaller inclusions. Current-voltage measurements showed that the resistivity of the CdMnTe wafer was reduced by $71 \%$, from $2.44 \times 10^{5} \Omega$-cm to $7.17 \times 10^{4} \Omega$-cm after annealing in Cd vapor.
\end{abstract}

Keywords: CdMnTe, defects, gamma-rays, nuclear and radiological detection, thermal annealing, X-rays.

\section{Introduction}

Room-temperature semiconductor nuclear detectors such as cadmium telluride (CdTe) and cadmium zinc telluride $(\mathrm{CdZnTe})$ could be operated without cryogenic cooling. This gives them the advantages of reduced operation cost and fabrication of portable and hand-held devices for the detection of X-rays and gamma-rays. CdTe and CdZnTe are commercially available [1]-[4], but the cost of their production is very high due to low growth yields of crystals with a high-resistivity, less defects and high-intrinsic efficiency. Recently, researchers have been investigating cadmium manganese telluride $(\mathrm{CdMnTe})$ for room-temperature nuclear and radiological detection applications [5]-[10]. The main advantage of growing CdMnTe crystal is that the segregation coefficient of $\mathrm{Mn}$ in cadmium telluride (CdTe) matrix is about 1.0, compared to $1.35 \mathrm{for} \mathrm{Zn}$ in the case of CdZnTe [9]. This is expected to result in the growth of CdMnTe with a uniform Mn content, which potentially would give a higher homogeneity and yield of detector-grade crystals [9], [10]. CdMnTe also has a wide energy band gap ( 1.7 to $2.2 \mathrm{eV}$ ), giving it a wide range of tenability, due to the compositional effect of $\mathrm{Mn}$ that increases it by $\sim 13 \mathrm{meV}$ per atomic percent of $\mathrm{Mn}$, compared to $6.7 \mathrm{meV}$ for $\mathrm{Zn} \mathrm{[10]-[12].}$

A major problem with CdMnTe crystals, also common to CdZnTe [13], is the presence of $\mathrm{Te}$ inclusions and related defects that limit their ability to detect X-rays and gamma-rays. In CdMnTe crystals grown by the Bridgman technique, the Te inclusions are formed from the evaporation of $\mathrm{Cd}$ and decomposition of segregated $\mathrm{Te}$ from the decomposition of the melt. One method of removing these performance-limiting $\mathrm{Te}$ inclusions is post-growth thermal annealing. There are two primary approaches to post-growth thermal annealing [14]: 1) annealing the crystal at a constant temperature (around or above the melting point of Te) in a $\mathrm{Cd}$ vapor, and 2) annealing under a temperature gradient to induce the migration of Te inclusions to the hightemperature side of the crystal. In temperature gradient annealing the migration of Te particles leaves regions with fewer and smaller Te inclusions, which can be harvested for detector fabrication. In constant temperature annealing in Cd-rich atmosphere, the $\mathrm{Cd}$ diffuses into the $\mathrm{CdMnTe}$ matrix where it combines with excess $\mathrm{Te}$ to form crystalline material. In the present work, we investigate how annealing in $\mathrm{Cd}$ vapor affects $\mathrm{Te}$ inclusions and the electrical resistivity of CdMnTe crystals. 


\section{Experiment}

CdMnTe crystal grown by Bridgman technique was cut from the ingot. The size of the CdMnTe wafer used in this experiment is $4.5 \mathrm{~mm} \times 4.0 \mathrm{~mm} \times 1.5 \mathrm{~mm}$ as measured using a digital Vernier caliper. The CdMnTe sample has medium resistivity of the order of $10^{5} \Omega-\mathrm{cm}$ (a detector-grade CdMnTe wafer is expected to have resistivity of the order of $10^{12} \Omega$-cm). The sample was mechanically polished with 800 -grit and 1200 -grit silicon carbide abrasive papers. This was followed by polishing on multi-tex pads with alumina powder of decreasing size from 3 microns to 0.1 micron. Infrared (IR) transmission microscopy was used to image the size and location of Te inclusions before and after annealing using AmScope Microscope. The AmScope Camera MD900E was adjusted to record IR images. The CdMnTe matrix is transparent to IR light while the Te inclusions are opaque and thus their images are recorded.

Current-voltage (I-V) measurements were carried out to study the effect of thermal annealing on the electrical resistivity of the CdMnTe sample. Before annealing, electrical contacts were deposited on the sample by depositing a gold chloride $\left(\mathrm{AuCl}_{3}\right)$ solution on the center of the two opposite planar surfaces of the $\mathrm{CdZnTe}$ wafer (see Fig. 1) using the electroless pipette method. After leaving the $\mathrm{AuCl}_{3}$ solution on a planar surface for about to 30 seconds, the excess solution was removed by blotting it out using the tip of a clean wiping cloth. The bulk leakage current for every applied voltage were measured for each sample using a Keithley Picoammeter/Voltage Source. The electrical contacts were removed before annealing and the samples polished in with alumina powder.

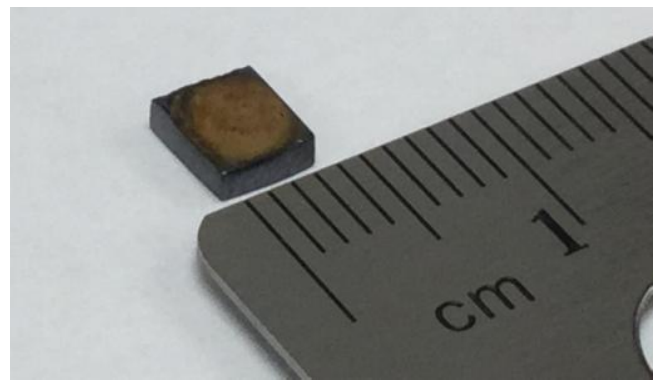

Fig. 1. CdMnTe wafer showing of the two circular-shaped gold contacts.

To anneal the sample, it was sealed in a quartz ampoule under a vacuum of $10^{-5} \mathrm{mbar}$ using the vacuum pump shown on the right-side of Fig. 2. The CdMnTe wafer was placed at one end of the ampoule and Cd was placed at the opposite end with a constriction between them. The three-zone furnace, shown on the left-side of Fig. 1, was used for annealing the samples. The heating temperatures of the heating elements were adjusted to get the desired temperature profile of the furnace to meet the desired annealing conditions. The temperature profile, shown in Fig. 3, was obtained by using a thermocouple placed and moved along the central axis of the cylindrical of the annealing furnace.

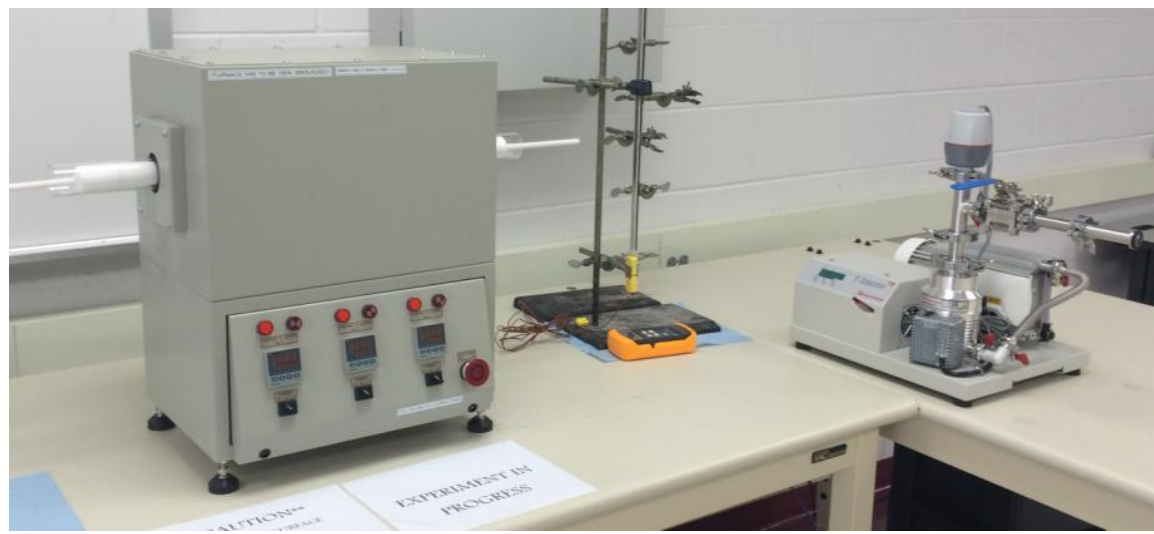

Fig. 2. Three-zone annealing furnace (shown on left-side) and vacuum pump (shown on right-side) for removing when sealing CdMnTe wafers in ampoules.

The three heating elements of the furnace enabled us to get constant temperature region of $720{ }^{\circ} \mathrm{C}$ where the sealed ampoule with the sample and $\mathrm{Cd}$ were placed. The sample was annealed for 12 hours. The polishing process, infrared transmission microscopy, electrical contacts deposition, and current-voltage measurements were repeated after annealing the sample. 


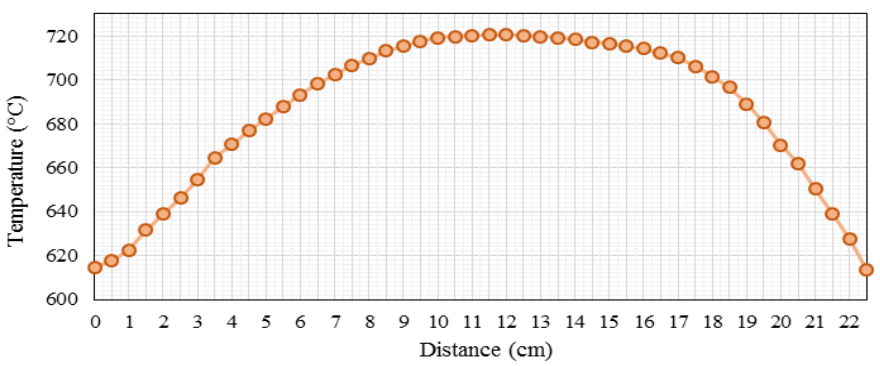

Fig. 3. The temperature profile of the furnace preset for annealing the CdMnTe samples.

\section{Results and Discussions}

The infrared images of the same region of the CdMnTe wafer before and after annealing in $\mathrm{Cd}$ vapor are shown in Fig. 4. There are changes in the shapes and sizes of the Te inclusions. The change in shape is more noticeable in the bigger Te inclusions. There is reduction in the dimension of the medium-size Te inclusion, located in the central right region. Some of the smaller inclusions in the upper side of the image have been eliminated while other were replaced with much smaller inclusions.

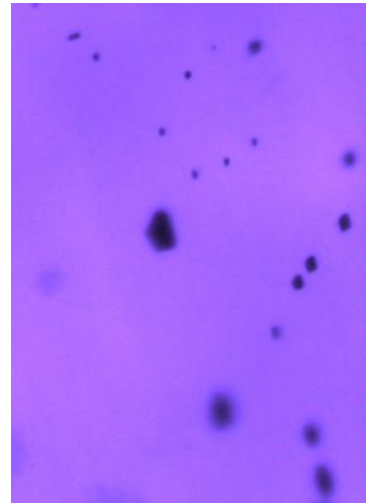

(a) Before annealing $(256 \mu \mathrm{m} \times 367 \mu \mathrm{m})$

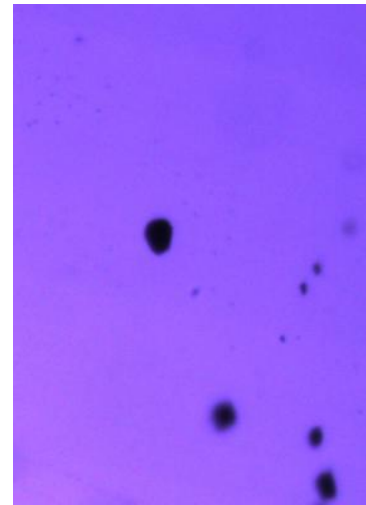

(b) After annealing $(256 \mu \mathrm{m} \times 367 \mu \mathrm{m})$

Fig. 4. Infrared images of the same region of the CdMnTe wafer before and after annealing in Cd vapor.

The change in shape, reduction in size, and elimination could be attributed to two major processes [14], viz. reaction between Te inclusions and $\mathrm{Cd}$ that diffused into the CdMnTe matrix, and diffusion of Te within the matrix. In the present work, we also observe a group of tiny Te inclusion that appeared in the CdMnTe wafer after annealing. This could be from Te particles diffusing from Te inclusions that were originally present in that region before annealing.

The current-voltage curves of the CdMnTe wafer before and after annealing are shown in Fig. 5. The resistivity $\rho$ of the material was calculated as

$$
\rho=\frac{R A}{l}
$$

where $R$ is electrical resistance, $A$ is contact area, and $l$ is the thickness of the CdMnTe wafer. Since $V=I R$, the resistance is obtained from the inverse of the slope on the linear portion of the I-V curves in Fig. 5 as

$$
R=\frac{\Delta V}{\Delta I}=\frac{1}{\text { slope }}
$$

From Eq. (1) and the slopes in Fig. 5 the resistivity of the CdMnTe wafer before annealing was found to be $2.44 \times 10^{5} \Omega-\mathrm{cm}$, and the resistivity after annealing in $\mathrm{Cd}$ vapor is $7.17 \times 10^{4} \Omega$-cm (the slopes were obtained using points corresponding to $21 \mathrm{~V}$ and $40 \mathrm{~V}$ ). This is a $71 \%$ reduction in the resistivity of the CdMnTe wafer. This is close to a reduction of one order of magnitude in the resistivity. In CdZnTe annealed at $670{ }^{\circ} \mathrm{C}$ for 50 hours in $\mathrm{Cd}$ vapor, it was reported that the resistivity of the material was reduced up to five orders of magnitude [15]. The reasons for these observations are changes caused by the annealing processes to the concentrations of deep-level Te anti-sites, $\mathrm{Cd}$ interstitials, and $\mathrm{Cd}$ vacancies, that are responsible for the high resistivity of CdZnTe [15]-[17], and in CdMnTe. For CdZnTe annealed in Cd vapor, it was suggested by Yang et al. [13] that the electrical compensation was lost due to extra $\mathrm{Cd}$ interstitials from the $\mathrm{Cd}$ vapor, thus resulting in shifting of the Fermi level towards the bottom of the conduction band. The same explanation could be applied to CdMnTe annealed in $\mathrm{Cd}$ vapor in this study. 


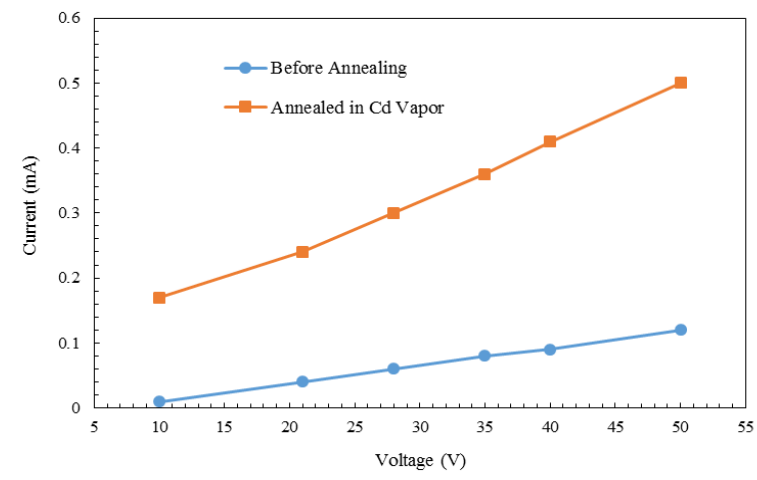

Fig. 5. Current-voltage curves of the CdMnTe wafer before and after annealing in $\mathrm{Cd}$ vapor.

\section{Conclusions}

We have presented experimental results of annealing a CdMnTe wafer, at $720^{\circ} \mathrm{C}$ and a vacuum of $10^{-5}$ mbar, in cadmium vapor. We used a three-zone furnace with three heating elements that enabled the adjustment of the temperature profile to get a flat region of $720{ }^{\circ} \mathrm{C}$ where the CdMnTe wafer was annealed, in a sealed quartz ampoule. Infrared transmission microscopy showed reduction in the dimension of the medium-size $\mathrm{Te}$ inclusions. Some Te inclusions were completely eliminated while others broke up to form much smaller inclusions.

The current-voltage measurements indicated that the resistivity of the CdMnTe crystal was reduced by $71 \%$, from $2.44 \times 10^{5} \Omega$-cm to $7.17 \times 10^{4} \Omega$-cm after annealing in $\mathrm{Cd}$ vapor. Detector-grade CdMnTe and CdZnTe wafers are expected to have resistivity of the order of $10^{12} \Omega-\mathrm{cm}$. The resistivity of CdMnTe crystals could be increased during crystal growth by doping the material with indium [1]. The problem of reduction in resistivity after annealing CdZnTe in Cd vapor could be resolved by annealing in a combination of $\mathrm{Cd}$-rich and $\mathrm{Zn}$-rich atmosphere [15]. Thus, it will be worth investigating the annealing of CdMnTe wafers in a combination of $\mathrm{Cd}$ and $\mathrm{Mn}$ vapors. The reduction in the resistivity of the CdMnTe wafer annealed in this study for 12 hours at $720{ }^{\circ} \mathrm{C}$ is within one order of magnitude. Reduction in resistivity of CdZnTe annealed at $670{ }^{\circ} \mathrm{C}$ for 50 hours in Cd vapor was reported to be up to five orders of magnitude. Thus, annealing for a shorter period (12 hours in this study) could be an advantage when compared to longer annealing periods.

\section{Acknowledgements}

This work has been supported by the U.S. Department of Homeland Security, Domestic Nuclear Detection Office, under competitively awarded contract/IAA award number 2012-DN-077-ARI065-05, and the U.S. Nuclear Regulatory Commission through award number NRC-27-10-514.

\section{References}

[1] S. U. Egarievwe, W. Chan, K.-H. Kim, U. N. Roy, V. Sams, A. Hossain, A. Kassu, R. B. James. "Carbon Coating and Defects in CdZnTe and CdMnTe Nuclear Detectors," IEEE Transactions on Nuclear Science, 63(1), 2016, $236-245$.

[2] N. Dedek, R. D. Speller, P. Spendley, and J. A. Horrocks, "Performance Evaluation of 98 CZT Sensors for Their Use in GammaRay Imaging," IEEE Transactions on Nuclear Science, 55(5), 2008, 2689-2697.

[3] H. Chen, S. A. Awadalla, P. Marthandam, K. Iniewski, P. H. Lu, G. Bindley, "CZT device with improved sensitivity for medical imaging and homeland security applications," Proc. SPIE 7449, Hard X-Ray, Gamma-Ray, and Neutron Detector Physics XI, San Diego, CA, 2009, 744902.

[4] S. A. Awadalla, J. Mackenzie, H. Chen, B. Redden, G. Bindley, M. C. Duff, A. Burger, M. Groza, V. Buliga, J. P. Bradley, Z. R. Dai, N. Teslich, D. R. Black, "Characterization of detector-grade CdZnTe crystals grown by traveling heater method (THM)," Journal of Crystal Growth, 312(4), 2010, 507-513.

[5] U. N. Roy, G. S. Camarda, Y. Cui, G. Gu, R. Gul, b, A. Hossain, G. Yang, S. U. Egarievwe, R. B. James. "Growth and characterization of CdMnTe by the vertical Bridgman technique," Journal of Crystal Growth, 437, 2016, 53-58.

[6] L. A. Mycielski, I. M. Rarenko1, T. Aoki, V. M. Sklyarchuk, O. L. Maslyanchuk, N. S. Yurtsenyuk, and Z. I. Zakharuk, "Energy band gap and electrical conductivity of $\mathrm{Cd}_{1-\mathrm{x}} \mathrm{Mn}_{\mathrm{x}}$ Te alloys with different manganese content," Semiconductor Physics, Quantum Electronics \& Optoelectronics, 14(4), 2011, 421-426.

[7] R. Rafiei, M. I. Reinhard, K. Kim, D. A. Prokopovich, D. Boardman, A. Sarbutt, G. C. Watt, A. E. Bolotnikov, L. J. Bignell, and R. B. James, "High-Purity CdMnTe Radiation Detectors: A High-Resolution Spectroscopic Evaluation," ," IEEE Transactions on Nuclear Science, 60(2), 2013, 1450-1456.

[8] Y. Du, W. Jie, X. Zheng, T. Wang, X. Bai, H. Yu, "Growth interface of In-doped CdMnTe from Te solution with vertical Bridgman method under ACRT technique", Trans. Nonferrous Met. Soc. of China, 22, 2012, s143-s147.

[9] A. Hossain, Y. Cui, A. E. Bolotnikov, G. S. Camarda, G. Yang, D. Kochanowska, M. Witkowska-Baran, A. Mycielski and R. B. James, "Vanadium-doped cadmium manganese telluride crystals as X- and gamma-ray detectors," Journal of Electronic Materials, 38, 2009, 1593-1599.

[10] Y. Du, W. Jie, T. Wang, Y. Xu, L. Yin, Pe. Yu, Ga. Zha, "Vertical Bridgman growth and characterization of CdMnTe crystals for gamma-ray radiation detector," Journal of Crystal Growth, 318, 2011, 1062-1066. 
[11] J. Zhang, L. Wang, J. Min, K. Qin, J. Huang, K. Tang, X. Liang, P. Shen, “Annealing of indium-doped CdMnTe single crystals under Cd vapors," Journal of Crystal Growth, 358, 2012, 12-15.

[12] L. A. Mycielski, I. M. Rarenko1, T. Aoki, V. M. Sklyarchuk, O. L. Maslyanchuk, N. S. Yurtsenyuk, and Z. I. Zakharuk, "Energy band gap and electrical conductivity of Cd1-xMnxTe alloys with different manganese content," Semiconductor Physics, Quantum Electronics \& Optoelectronics, 14(4), 2011, 421-426.

[13] A. E. Bolotnikov, S. Babalola, G. Camarda, Y. Cui, S. U. Egarievwe, R. Hawrami, A. Hossain, G. Yang, R. B. James, "Te Inclusions in CZT Detectors: New Method for Correcting Their Adverse Effects," IEEE Transactions on Nuclear Science, 57(2), 2010, 910-919.

[14] S. U. Egarievwe, G. Yang, A. A. Egarievwe, I. O. Okwechime, J. Gray, Z. M. Hales, A. Hossain, G. S. Camarda, A. E. Bolotnikov, and R. B. James, "Post-Growth Annealing of Bridgman-Grown CdZnTe and CdMnTe Crystals for Room-Temperature Nuclear Radiation Detectors." Nuclear Instrumentation and Methods in Physics Research A, 784, 2015, 51-55.

[15] G. Yang, A. E. Bolotnikov, P. M. Fochuk, Y. Cui, G. S. Camarda, A. Hossain, K. H. Kim, J. Horace, B. McCall, R. Gul, O. V. Kopach, S. U. Egarievwe, R. B. James, "Post-growth Annealing of Cadmium Zinc Telluride Crystals for Room-Temperature Radiation Detectors," Journal of Electronic Materials, 41(10), 2012, 2912-2916.

[16] V. Babentsov, J. Franc, P. Hoschl, M. Fiederle, K. W. Benz, N. V. Sochinskii, E. Dieguez, and R. B. James, "Characterization of compensation and trapping in CdTe and CdZnTe: Recent advances," Cryst. Res. Technol., 44(10), 2009, 1054-1058.

[17] M. Fiederle, C. Eiche, M. Salk, R. Schwarz, K. W. Bens, W. Stadler, D. M. Hofmann, and B. K. Meyer, "Modified compensation model of CdTe," Journal of Applied Physics, 84(12), 1998, 6689-6692. 